\title{
An Improved Fast Motion Estimation Algorithm Based on UMHexagonS
}

\author{
Meng Wang ${ }^{1, a^{*}}$, Xia Zhang ${ }^{2, b}$ \\ ${ }^{1}$ Information Engineering School, Communication University of China, Beijing, China \\ ${ }^{2}$ Information Engineering School, Communication University of China, Beijing, China \\ awang_meng@cuc.edu.cn, bzhangxia@cuc.edu.cn
}

Keywords: video compression; H.264/AVC; motion estimation; UMHexagonS; search template; center bias

\begin{abstract}
Motion estimation plays an important role in H.264 video compression process. However, it takes $60 \%-80 \%$ of the encoding time. In this paper, an improved motion estimation algorithm based on UMHexagonS algorithm is proposed, including three aspects which are improvement on unsymmetrical-cross search, improvement on $5 \times 5$ square search and improvement on Multi-Hexagon-grid search. Experimental results show that the proposed algorithm has better performance compared with the original algorithm, which reduces the encoding time without much PSNR degradation and bit-rate increasing.
\end{abstract}

\section{Introduction}

The H.264 [1] video coding standard proposed by Video Coding Expert Group (VCEG) of ITU-T and Moving Picture Experts Group (MPEG) of ISO/IEC has more better coding efficiency and more better video quality than many other video coding standard. However, the cost of high performance in video compression coding is great computational complexity. In other word, it will need more time and more software or hardware source for video compression. And motion estimation takes $60 \%-80 \%$ of the encoding time [2].

So, it is necessary that reducing the complexity and the time of motion estimation. In order to achieve this goal, many experts provided many fast motion estimation algorithms, such as Four-step search algorithm(FSS) [3], New Three Step Search [4], Diamond search algorithm(DS) [5], Hexagon search algorithm(HS) [6], etc. Compared to the Full Search Algorithm (FS), Unsymmetrical-cross Multi-hexagon-grid Search algorithm ( UMHexagonS ) which combines many search techniques can save $90 \%$ computations. And at the same times, it keeps the quality of video. But, there still are some defects in UMHexagonS algorithm.

An improved motion estimation algorithm which is based on UMHexagonS algorithm is proposed in this paper. The improved motion estimation algorithm includes improvement on unsymmetrical-cross search, improvement on $5 \times 5$ square search and Multi-Hexagon-grid search.

\section{Description of the UMHexagonS Algorithm}

Idea of the UMHexagonS. The full name of the UMHexagonS algorithm is Unsymmetrical-cross Multi-Hexagon-grid Search algorithm. It combines many kinds of search template and early termination algorithms to reduce motion estimation time. Because of the combination of many fast search techniques, the algorithm performance is better than many other fast motion estimation algorithms.

Analysis. Although the UMHexagonS algorithm uses many methods to improve the accuracy and to reduce search time, the high coding efficiency based on some new techniques also leads to high computational complexity. For the algorithm is too complex, it will influence the encoding time. So, we try to find a balance between the computational complexity and encoding time in this paper.

The searching strategy which finds optimum motion vector point by point contributes little to improve the accuracy of motion estimation, and causing a waste of search time. On the basis of the 
video sequence features, the proposed algorithm reduces the number of the search point in the motion estimation process. From the experimental results, the proposed algorithm simplifies the complexity and reduces the encoding time. Meanwhile, the algorithm keeps the quality of test video sequences.

\section{Improving the Process of UMHexagonS Algorithm}

Improvement on the Unsymmetrical-cross Search Template. It is apparent that the motion vector of most natural video sequences has characteristic of center bias [7]. According to the characteristic, we will improve the unsymmetrical-cross template.

Reference [7] has used the idea of the characteristic of motion vector, and proposed a new unsymmetrical-cross template, which effectively reducing motion estimation time. Difference increase series is that the difference between the two numbers ascends by natural numbers. And the difference increase series conforms to the characteristic of center bias in video sequence. The new search template uses difference increase series $1,2,4,7,11 \ldots$ as the horizontal and vertical coordinates of search template. Naturally, the same is true in the negative axis. And the points which are out of the search range will be abandoned. The search template is depicted in Fig. 1.

In fact, we can use another difference increase series to reduce the number of the search point. In this paper, we use difference increase series $1,3,6,10,15 \ldots$ instead of the above difference increase series, as Fig. 2. Quite obviously, the number of the new search template is not more than that of the search template in the reference.

After test, we find there are not much PSNR degradation and bit-rate increasing. On the contrary, it will get a better performance sometimes.

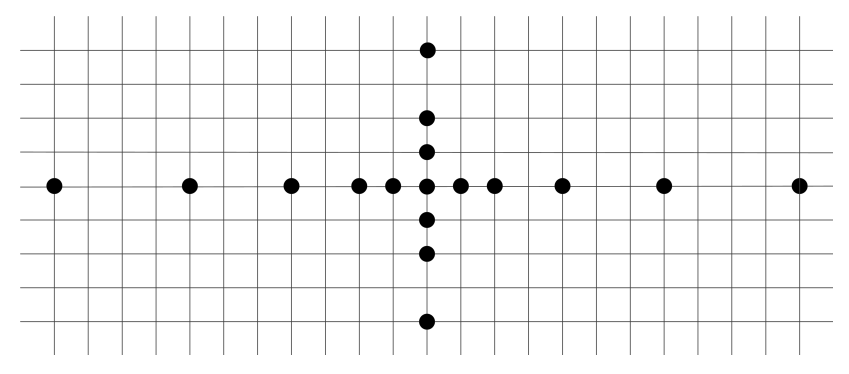

Fig. 1 Proposed template in reference

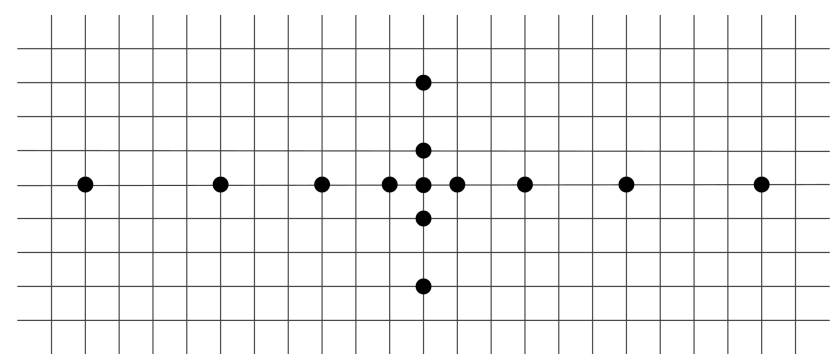

Fig. 2 New unsymmetrical-cross template in this paper

Improvement on $5 \times 5$ Rectangular Full Search Template. In the original UMHexagonS algorithm, $5 \times 5$ rectangular full search needs to search 24 points every time, as Fig. 3. The computational complexity is great and it will takes more time on encoding. Chi-Wai Lam, Lai-Man Po and Chun Ho Cheung note that more than $80 \%$ of the MV predicted value distribute in $5 \times 5$ rectangle area, and provide the probability of the MV predicted value about every point [8]. According to the literature, we adopt a new search template to reduce computational complexity. The new search template is shown in Fig. 4. This improvement effectively reduces the motion estimation time without PSNR degradation and bit-rate increasing. 


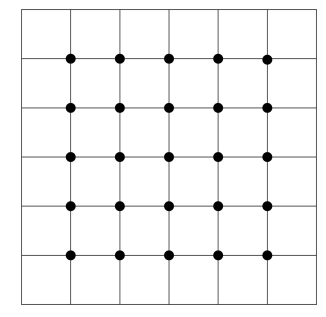

Fig. $35 \times 5$ square

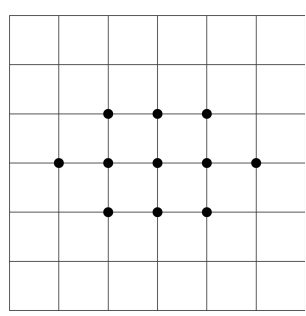

Fig. 4 New search template

The new search template proposed in Fig. 4, provides a pretty good performance. According to the reference [8], more than $70 \%$ of the MV predicted value distribute in the hexagon area shown in Fig. 4. The hexagon area also conforms the characteristic of MV. After using the new search template, we can put 24 search points down to 10 points, it is decreased by 58\%. But at the same time, the quality of test sequences has no much degradation. In our test, we used six QCIF test sequences: Akiyo, Carphone, Coastguard, Container, Foreman, Highway. The simulation results show that the quality of test sequence named coastguard has degradation, while other test sequences keep a good or a better quality.

On the other hand, the final step in UMHexagonS algorithm is search points by small diamond template. Consequently, many points will be searched several times. So, we can further reduce the number of the search points. We use the uneven hexagon search template instead of the original $5 \times 5$ rectangle template, as Fig. 5 . The template will search 6 points. Compared with original $5 \times 5$ rectangle search template, the number of the search points is decreased by $75 \%$. It is obvious that the uneven hexagon search template will reduce motion estimation time significantly. In the meantime, we find it keeps the quality of the test sequences after simulations. We also uses the above six test sequences, the results shows that there are not much degradation in PSNR and bit-rate increasing. The new search template offers similar performance in motion estimation process.

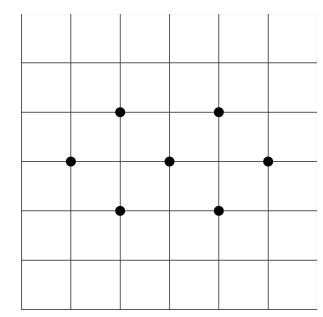

Fig. 5 Uneven hexagon search template

Improvement on Multi-level Hexagons Template. In step3, multi-level hexagons search needs to search 16 points in each layer. After searching all of the 16 points in one layer, the algorithm will determine whether to go to the Early Termination. In many cases, we have to check all of the 64 positions of the four hexagons.

Taking into account the fact that the motion vector of the video sequence has characteristic of center bias, the paper proposes a new search template. First, the algorithm searches 16 points, after this step searches 12 points. The search template is shown as Fig. 6.

In the first step, the hexagon template keeps a good accuracy in motion estimation process. The multi-level octagon search template is more similar to the circle and more symmetrical in all directions.

The experimental test of this search template shows good performance. Therefore, this paper selects this new search template instead of the original template. 


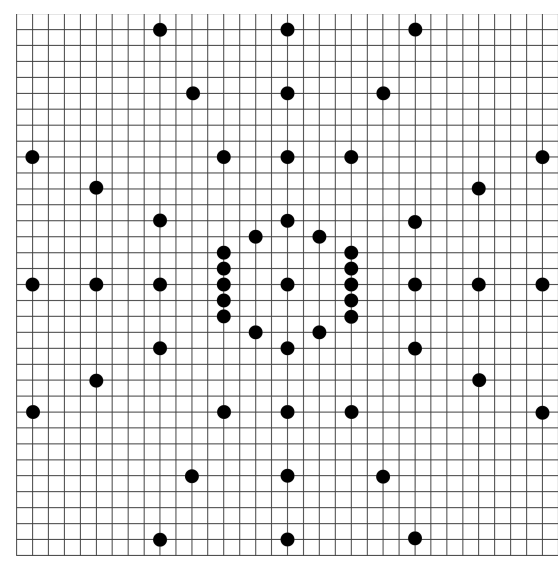

Fig. 6 Hexagon and multi-level octagon hybrid template

\section{Experimental Results and Analysis}

We modify the program in JM18.6 which is the reference software of H.264/AVC to implement the new algorithm proposed in this paper. The experimental platform is: Windows7 system, Intel(R) Core(TM)i3-390M@2.66GHz RAM 2.00GB. The coding environment is Microsoft Visual Studio 2010.

Experiments parameter configuration is shown as follows:

SourceWidth $=176$, SourceHeigh $=144$, FramesToBeEncoded $=30$, FrameRate $=30.0$, RDOptimization $=1$, IntraPeriod $=0$, SearchRange $=32$, NumberReferenceFrames $=5$.

And other parameters keep the default values. Simulation uses six QCIF test sequences: Akiyo, Carphone, Coastguard, Container, Foreman, Highway, because these six test sequences have different motion intensity.

The experimental results are shown in TABLE I, TABLE II and TABLE III.

TABLE I suggests that the improved algorithm in this paper reduces the motion estimation time effectively. In the best case, it can reduce about $20 \%$ motion estimation time compared to the original algorithm. In the meantime, the PSNR just degrades $0.013 \mathrm{~dB}$ and the bit-rate increases less than 0 . $4 \%$ on average. It is obvious that the improved algorithm search lesser points than original algorithm. It will takes less time to finish the motion estimation. But the rest points provide enough information to keep the quality of these test video sequences.

Table 1 Results of Motion Estimation Time

\begin{tabular}{|c|c|c|c|}
\hline \multirow{2}{*}{$\begin{array}{c}\text { Video } \\
\text { Sequence }\end{array}$} & \multicolumn{3}{|c|}{ Motion Estimation Time[sec] } \\
\cline { 2 - 4 } & UMHexagonS & $\begin{array}{c}\text { Improved } \\
\text { algorithm }\end{array}$ & $\begin{array}{c}\text { Change } \\
\text { Rate }\end{array}$ \\
\hline akiyo & 24.562 & 20.082 & $-18.24 \%$ \\
\hline carphone & 27.315 & 27.027 & $-1.05 \%$ \\
\hline coastguard & 47.022 & 42.394 & $-9.84 \%$ \\
\hline container & 24.095 & 21.018 & $-12.77 \%$ \\
\hline foreman & 38.881 & 33.986 & $-12.59 \%$ \\
\hline highway & 33.223 & 26.127 & $-21.36 \%$ \\
\hline
\end{tabular}

Table 2 Results of PSNR-Y

\begin{tabular}{|c|c|c|c|}
\hline \multirow{2}{*}{$\begin{array}{c}\text { Video } \\
\text { Sequence }\end{array}$} & \multicolumn{3}{|c|}{ PSNR-Y[dB] } \\
\cline { 2 - 4 } & UMHexagonS & $\begin{array}{c}\text { Improved } \\
\text { algorithm }\end{array}$ & $\begin{array}{c}\text { Change } \\
\text { Rat }\end{array}$ \\
\hline akiyo & 38.559 & 38.578 & +0.019 \\
\hline
\end{tabular}




\begin{tabular}{|c|c|c|c|}
\hline carphone & 37.473 & 37.477 & +0.004 \\
\hline coastguard & 34.522 & 34.509 & -0.013 \\
\hline container & 36.426 & 36.426 & 0 \\
\hline foreman & 36.855 & 36.880 & +0.025 \\
\hline highway & 37.992 & 37.999 & +0.007 \\
\hline
\end{tabular}

Table 3 Results of Bit-rate

\begin{tabular}{|c|c|c|c|}
\hline \multirow{2}{*}{$\begin{array}{c}\text { Video } \\
\text { Sequence }\end{array}$} & \multicolumn{3}{|c|}{ Bit-rate[kbps] } \\
\cline { 2 - 4 } & UMHexagonS & $\begin{array}{c}\text { Improved } \\
\text { algorithm }\end{array}$ & $\begin{array}{c}\text { Change } \\
\text { rate }\end{array}$ \\
\hline akiyo & 37.36 & 37.75 & $+1.04 \%$ \\
\hline carphone & 119.86 & 120.99 & $+0.94 \%$ \\
\hline coastguard & 233.98 & 234.30 & $+0.14 \%$ \\
\hline container & 50.78 & 50.78 & 0 \\
\hline foreman & 145.67 & 146.06 & $+0.27 \%$ \\
\hline highway & 77.23 & 76.90 & $-0.43 \%$ \\
\hline
\end{tabular}

\section{Conclusion}

In this paper, an improved algorithm based on the UMHexagonS algorithm proposed in order to reduce the complexity of motion estimation and keep the quality of video. The experiment shows that the proposed algorithm reduces the computational complexity of motion estimation and is highly effective in reducing motion estimation time from 10-20\%. Thus the proposed algorithm improves the performance of H.264/AVC encoding effectively.

\section{References}

[1] T. Wiegand, G. J. Sulivan, and A. Luthra. "Draft ITU-T Recommen- dation H.264 and Final Draft International Standard 14496-10 AVC”. JVT of ISO/IEC JTC1/SC29/WG11 and ITU-T SG16/Q. 6, Doc. JVT-G050r1, Geneva, Switzerland, May 2003.

[2] Yufeng Li, Jufei Xiao, Wei Wu. "Motion Estimation Based On H.264 Video Coding”, International Congress on Image and Signal Processing(CISP), 2012 International Conference on. IEEE, 2012.

[3] Lai-Man Po and Wing-Chung Ma. "A novel four-search algorithm for fast block motion estimation”. IEEE Transactions on Circuits and Systems for Video Technology, 1996, 6(3): 313-317.

[4] Li Reoxiang, Zeng Bing, M.L. Liou. "A new three-step search algorithm for block motion estimation”. IEEE Transactions on Circuits and Systems for Video Technology,1994,4(4):438-442.

[5] Shan Zhu and Kai-Kuang Ma, “A new diamond search algorithm for fast block matching motion estimation”. IEEE Trans-Image Processing.2000, 9(2):287-290.

[6] Ce Zhu, Xiao Lin and Lap-Pui Chau. "Hexagon based search pattern for fast block motion estimation”. IEEE Transactions on Circuits and System for VideoTechnology,2002,12(5):349-355.

[7] Liu Chen, Jian-zhong Cao, Li-nao Tang, Ji-Jiang Huang, Hui-nan Guo and Xiao-kun Dong. “Optimized H.264 Motion Estimation Algorithm Based on UMHexagonS”, International Conference on Intelligent Control and Information Processing(ICICIP),2013 International Conference on. IEEE, 2012.

[8] Chi-Wai Lam, Lai-Man Po and Chun Ho Cheung. "A novel kite-cross-diamond search algorithm for fast block motion estimation," IEEE International Symposium on Circuits and Systems, vol. 3, pp 729-732, 2004. 